832; 1971) may attract a large audience. In the past, attempts to isolate eukaryotic messengers have involved exposing polysome preparations to either detergents or chelating agents. The former procedure releases all the RNA species in polysomes and leaves the problem of separating the messenger RNA from the transfer, 5S and ribosomal RNAs. The latter procedure results in the release only of the messenger and some transfer RNA but the messenger is found complexed with protein. To avoid these problems Blobel has developed a recipe for treating polysomes with puromycin at high ionic strengths, the net result of which is the dissociation of the ribosomes and the release of naked messenger and transfer RNAs which are readily separated. Using this procedure Blobel obtains from rabbit reticulocyte polysomes an RNA which sediments at 11.3 $\mathrm{S}$ whereas the other procedure for isolating globin messenger from these polysomes yields a 9S RNA. A simple calculation suggests that this $11.3 \mathrm{~S}$ RNA may have about 230 bases in addition to the 450 -odd bases needed to code for globin. It would be no surprise if these additional bases formed sequences at the $5^{\prime}$ and/or $3^{\prime}$ ends of the globin messenger which are not involved in coding, for that is what the partial sequences of the RNA phage RNAs lead us to expect. And how widely applicable is this puromycin procedure? Although Blobel has worked chiefly with polysomes he says that it may be generally useful.

The basis of Blobel's procedure is, of course, the reaction between peptidyltRNA and puromycin-an analogue of aminoacyl-tRNA - to yield free peptidyl-puromycin. Raacke (Biochem. Biophys. Res. Comm., 43, 168; 1971) has constructed space filling models of puromycin and peptidyladenylic acid (to represent the terminus of peptidyltRNA) to investigate the stereochemistry of this reaction. In short he finds puromycin can assume a Ushaped configuration and "hug" the terminal adenine residue of the peptidyl-tRNA such that the amino-group of the antibiotic is favourably placed to react with the carbonyl group of the peptidyl-tRNA. All this apparently takes place at the peptidyl site of the ribosome to which the adenine of the peptidyl-tRNA is bound. Raacke envisages that the dimethyladenine of puromycin interposes itself between this adenine and its binding site. The puromycin is then bound to the peptidyl site and the peptidyl-tRNA is bound to the antibiotic by hydrophobic attraction of stacked bases, the dimethyladenine and methoxybenzene of the antibiotic alternating with the terminal adenine and penultimate cytosine of the peptidyl-tRNA.
CANCER

\section{Aggressive Lymphocytes}

from a Correspondent

THE notion that there exists a "natural" defence mechanism against the growth of malignant cells is deep rooted in the folklore of cancer research. The evidence in the distant past has been scanty, but reinforced by the will of those investigators who saw the possibility of manipulation of the immune mechanism as a means of control of cancer. Perhaps the biggest objection has always been that if there is a defence mechanism why does it fail so often and why do so many people die with cancer? To say that many of them smoke too much is irrelevant to the present argument.

The concept of the tumour specific antigen has been adduced largely from work with experimental animals and many studies have exemplified such antigens and attempted, in the main unsuccessfully, their characterization. But a recent article by the Hellströms and their associates (Intern. J. Cancer. 7,226 ; 1971) helps both to reconcile some previously conflicting ideas and to indicate the road ahead for those interested in tumour immunology.

The basic data, which derive largely from previous work of the Hellströms, are that the blood lymphocytes from human patients with a variety of tumours can specifically kill neoplastic cells, in vitro, from the same patients. In addition, in many instances cells from tumours of similar morphological types from other patients can also be killed. The failure of the aggressive lymphocytes effectively to reduce the malignant cell population in the host of origin has been attributed to the inhibitory effect of blocking antibodies. It is envisaged that in some way these antibodies, which are produced as part of the complex of immune processes, prevent the lymphocytes from reaching, recognizing or destroying their target cells.

The latest article tests the effect that serum from cancer patients can have in preventing lymphocytes from killing tumour cells in vitro. A high proportion of sera were effective and ostensibly specific in that they only blocked the reactions between lymphocytes and tumour cells which derived from patients with a similar tumour: host relationship to the serum donor. Hellström and his colleagues point out that their methods provide an in vitro index of the magnitude of both cellular and humoral components of the anticancer response in patients and they could eventually provide diagnostic and prognostic tools for the cancer therapist.

\title{
Long Term Variations of the Plasmapause
}

In the next issue of Nature Physical Science, J. Oksman of the University of Oulu, Finland, proposes annual and sunspot cycle variations in the position of the terrestrial plasmapause.

In the 1930 s it was realized that the magnetic field of the Earth could not simply be that of a dipole, because observations of the low frequency variations in the field intensity (micropulsations) showed the field to be compressed on the daylit side of the globe. In 1931, Chapman and Ferraro (Terr. Magn. Atmos. Elect., 36, 171; 1931) produced a simple model of this compression produced by the interplanetary flux of charged particles (plasma) flowing out from the solar corona.

The interaction of this flux, the solar wind, with the geomagnetic field has been studied intensively over the past decade. It has been shown that the plasma in the vicinity of the Earth is contained within a magnetospheric boundary defined by the solar wind velocity and particle density. By day the magnetosphere-the region within the boundary - has a dimension of order 10 Earth radii $\left(R_{E}\right)$ but at night the boundary is extended into a long magnetospheric "tail".

In the early 1960 s it was believed that the ionization density within the mag. netosphere showed a smooth fall off with increasing distance from the Earth. But in 1963 it was demonstrated, from an extensive analysis of the characteristics of audible frequency natural electromagnetic signals (whistlers), that there was a marked discontinuity or "knee" in plasma density at a distance, from the Earth, of the order $3 R_{\mathrm{E}}$ (D. $\mathrm{L}$. Carpenter, J. Geophys. Res., 68, 1675; 1963). Just within the knee the density is around 100 electrons per $\mathrm{cm}^{3}$ but outside the density it is only a few electrons per $\mathrm{cm}^{3}$. The high density region is now known as the plasmasphere and its boundary is termed the plasmapause.

Oksman proposes that the observed variations in the paths of whistlers and most of the observations of geomagnetic micropulsations in a particular frequency band, can be explained if it is assumed that there are annual and secular (sunspot cycle) changes in the size of the plasmasphere. The annual variation deduced is of order $1 R_{E}$ with a smaller secular variation. The motions, it is suggested, are linked with the changing tilt angle of the geomagnetic axis to the ecliptic plane, and Oksman believes that this factor may also explain ionospheric anomalies. 